Problem Solving in a Middle School Robotics Design Classroom

\author{
Stephen J. Norton, \\ Griffith University
}

Campbell J. McRobbie and Ian S. Ginns

Queensland University of Technology

Australia

Contact details:

Dr. Stephen Norton

Prof. Campbell McRobbie

Dr. Ian Ginns 


\title{
Problem Solving in a Middle School Robotics Design Classroom.
}

\begin{abstract}
Little research has been conducted on how students work when they are required to plan, build and evaluate artefacts in technology rich learning environments such as those supported by tools including flow charts, Labview programming and Lego construction. In this study, activity theory was used as an analytic tool to examine the social construction of meaning. There was a focus on the effect of teachers' goals and the rules they enacted upon student use of the flow chart planning tool, and the tools of the programming language Labview and Lego construction. It was found that the articulation of a teacher's goals via rules and divisions of labour helped to form distinct communities of learning and influenced the development of different problem solving strategies. The use of the planning tool flow charting was associated with continuity of approach, integration of problem solutions including appreciation of the nexus between construction and programming, and greater educational transformation. Students who flow charted defined problems in a more holistic way and demonstrated more methodical, insightful and integrated approaches to their use of tools. The findings have implications for teaching in design dominated learning environments.
\end{abstract}

The American Association for the Advancement of Science (1993), through Project 2061, actively promoted the inclusion of technology in the school curriculum and also recommended that technology could be used as a vehicle for learning science. This sentiment has been mirrored in Queensland curriculum documents (e.g., Education Queensland, 2001; Queensland School Curriculum Council, 1999; Queensland Studies Authority, 2003) that recommend the adoption of an integrated and a learnercentred approach to the teaching and learning of science, through which students actively construct meaning.

One tool that has had some support as a means for integrated teaching of science, mathematics and technology is Lego Robotics. Student learning via the construction of robots has evolved to a large degree from the work of Seymour Papert who coined the term "constructionism" and supported the use of Lego construction in conjunction with programming (Robotics) to develop understandings in mathematics and science as well as general problem solving and creativity (Papert \& Harel, 1991). The use of technology based learning environments has also found the support of Roth (2001).

Several studies (e.g., Bergen, 2001; Mauch, 2001; Noble, 2001) reported the strong motivational potential of working with Lego Robotics and in developing problem solving strategies. In addition, Levien and Rochefort (2002) commented on Lego Robotics as a suitable medium to explore engineering principles with tertiary students. Research into Lego Robotics in middle school years indicates that many opportunities for extracting science and mathematics principles from technology-based activities are not capitalised on, the science and mathematics remaining implicit (e.g., McRobbie, Norton, \& Ginns, 2003). A particular concern has been teachers' difficulties in making the links between the technology activity of Robotics and other syllabus outcomes. McRobbie et al. (2003) also showed how the programming feature of Lego Robotics (Labview) had the potential to absorb most of the students' problem 
solving endeavours and the science and mathematics associated with the construction and mechanical operation of the robots became a secondary concern of both students and teachers. Relatively little research has been conducted into students' approaches to design and construction (Oxman, 1999), in particular, in complex multimedia learning environments such as robotics.

The National Advisory Committee on Creative and Cultural Education (NACCCE1998) notes that technological and scientific creativity carries with it the idea of action and purpose, with drafting or giving an idea a rough shape or outline being the first phase of the creative process. In curriculum documents drafting is referred to as planning (e.g., Queensland Studies Authority, 2004). Planning is a cognitive activity where the development of an appropriate solution strategy begins and it is a process that students need to learn in order to problem solve (Deek, Hiltz, Kimmel, \& Rotter, 1999). It is a central activity embodied in curriculum documents in all three subject domains of science, mathematics and technology used in most western educational systems. A tool that is sometimes used in the problem solving process is the construction of a flow chart. In general, the research on flow chart construction indicates that it is a useful tool for enhancing understanding and decision making processes as well as assisting in the development of critical thinking skills (e.g., Crews \& Butterfield, 2003; Satchwell, 1997). Crews and Butterfield (2003) noted that the use of a flow charting based programming environment was a useful means to emphasise logic and Satchwell (1997) found that functional flow diagrams were effective at increasing the students' understanding at a foundational level as well as enhancing their ability to understand systems theory and to predict systems outcomes. Satchwell considered the simplicity of such diagrams, and their usefulness in conveying information about meaningful relationships between concepts and component parts, and causal relationships, were important attributes that could explain the success of flow charting as a tool to assist learning. However, the use of flow charting by middle school students as a planning tool, particularly in the context of programming in a robotics environment has not been documented.

It is well known that teachers' specific goals are an important influence upon their teaching orientation (Alexander, 1996) and teachers' beliefs about the nature of teaching are critical to their implementation of pedagogy and how students go about their work (e.g., Thompson, 1992). Thus, the aim of this study was to investigate the interactions between teachers' beliefs and pedagogical practices (teachers' activity) upon middle school students' approaches to designing, problem solving and constructing in a Lego Robotics environment (student activity).

\section{Design and Method}

This was an interpretive study (Erickson, 1998) that was informed by the criteria for excellence in qualitative research (Lincoln \& Guba, 2000). It involved the in-depth investigation of the implementation of a robotics unit of work for one semester (20 weeks) in a middle school located in the Brisbane metropolitan area. The robotics unit was studied by two composite classes comprised of Grade 8, 9 and 10 girls and boys.

The two teachers were George and Callum (pseudonyms) who developed and taught the unit. Neither teacher had taught robotics previously. George, a science teacher with over 25 years experience, specialised in teaching physics and had some computer programming expertise. Callum, a first year science teacher, was formerly an experienced design electrical engineer and had considerable expertise in programming. The classes contained a spread of student abilities and they worked in 
self-selected groups of three. Three groups of students in each class were selected for in-depth study, based on the advices from the respective teacher to represent variations in capability in science and problem solving. The group number, names of students (pseudontyms), and respective teacher are as follows.

In Callum's class - the following groups were:

Group 1, Year 8 boys: Laurie, Mike and Ron.

Group 2, Year 8 girls: Amy, Marilyn, and Cassy.

Group 3, Year 8 boys: Wil, Terrance, and Sylvester.

In George's class the following groups were focus groups:

Group 4, Year 8 boys: David; Matt, Bill and Dale.

Group 5, Year 10 girls: Cass, Steph, and Alli.

Group 6, Year 9 girls: Chantelle, Gem, and Sam

The robotics unit: The robotics unit focused on the programming, design and construction of robots to complete a CanDo Challenge problem solving task. The challenge required students to construct a robot of their own design, and program it to remove six weighted soft drink cans from a one metre diameter circle in a two minute time frame. The robot had to stop at the two minute time. The major problem to be overcome related to constructing powerful and reliable robots capable of pushing the cans, developing effective search patterns, programming the robots to return to the circle after pushing out cans and to stop after two minutes.

The principal instruments (tools) used by each group were (a) flow charts; (b) Labview, an icon based programming language loaded into a stand-alone computer; and (c) Lego construction tools comprised of a Lego RCX brick (computer) and Lego building components. The software was used to program RCX bricks, a central piece of each Robolab kit. A brick could be loaded with five computer programs, and had three input ports for the monitoring of any three of the variables light, touch, temperature and sound. Only touch and light sensors were used in the Can Do Challenge. Three output ports on the RCX brick were used mainly to drive motors. There were assorted Lego construction components including gears, wheels, axles, tracks, rods, and plates and connectors available to complete the physical construction of the robot. The principal teaching instruments the teachers used were the classroom space, the white board, the digital data projector and the robotics equipment.

Data sources: These included observations and videotapes of classroom interactions, videotapes of computer screen displays and students' activity, teacher and selected student interviews, and collected artefacts (programs, plans, reports and photographs of robots) throughout the semester.

Analysis: The analytical framework for describing how students worked was through the lens of activity theory (Leontyev, 1977; Engestrom \& Miettinen, 1999). The central aspect of activity theory is that the object connects individual actions to collective activity. Activity is always connected with the transformation of reality (Davyov, 1999) and recognises that two basic processes operate continuously; internalisation and externalisation. Internalisation is related to the reproduction of culture and externalisation to the creation of new artefacts that makes possible this transformation (Engestrom \& Miettinen, 1999). Activity theory places people as actors in cultural contexts, shaping and being shaped by the physical environment (Leontyev, 1977). This dual nature of "interaction" between mind and action is mediated by tools that include artefacts, language, symbols, rules and ideas. Tolman 
(1999) notes that in order to understand the activity in an individual's life we need to appreciate that activity is built up in the concrete conditions of life and this includes the social nature of the individual. This analytical framework helps to enable the reader to make sense of the activity taking place in the classroom. Frequently, models of activity theory show interacting triangles that include the nodes of Subjects, Instruments (Mediating artefacts, tools or signs), Rules, Community, Division of labour and Objects (e.g., Roth, Tobin, Zimmermann, Natasia, \& Davis, 2002) such as depicted in Figure 1. In this paper the Subjects refers to the teachers and the students. There is a focus on how the teachers' beliefs and goals relate to their pedagogy and the relationships between these and student activity. Rules refer to the explicit and implicit classroom regulations, norms and conventions that shape classroom activity; the Community refers to the classroom community at the macro level and the student groups at the micro level; Division of labour refers to the roles and task of teachers and students; Instruments or Mediating artefacts, tools or signs includes flow charting and also refers to the classroom equipment that includes Labview and Lego construction materials allocated to each group and the curriculum. Each of these attributes interact to produce Objects, in this study defined as learning outcomes, in particular the products and processes involved in students constructing and programming to have their robots perform the set challenges.

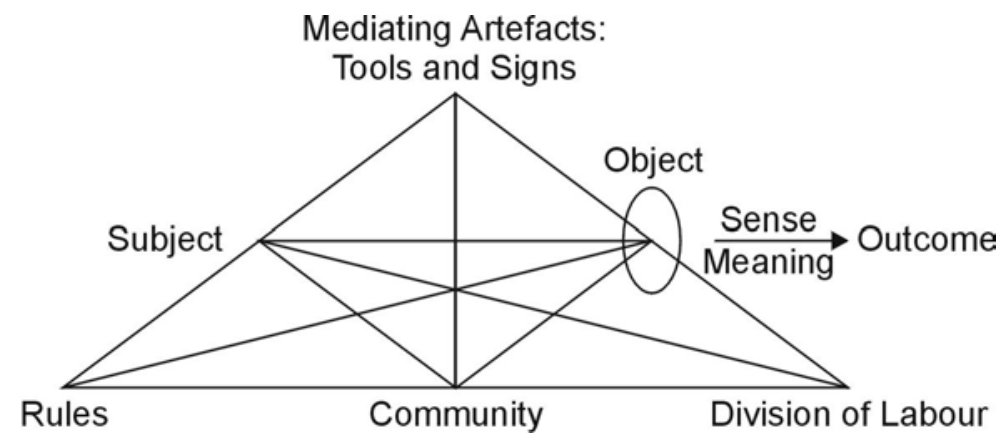

Figure 1: Model of Activity Theory.

\section{Results}

The results are presented in the form of assertions derived from the various sources of data. In this process, the beliefs and pedagogy of the teachers is discussed first, followed by the student activity. While all students were observed and interviewed, six groups were selected for more detailed observation throughout the life of the study.

Assertion 1: Teachers' goals and beliefs mediated the formation of different learning communities.

\section{Callum}

Callum articulated his goals as follows. He stated that his principal goal was to use the technology teaching unit to get students to think for themselves in a logical way. He stated:

I think it is great to get kids to think about how to logically do things rather than just learning things. In other subjects you can not get the teacher to be a facilitator, here we can get the kids to think for themselves rather than just being told. 
This statement is evidence that he saw students as the main source of information and favoured learning environments that lacked certainty. Callum identified programming and in particular planning as the central cognitive activity.

I have not been strong on the robotic design, the actual hardware because I have the approach that anyone can build a Lego thing. The intelligent part is the programming....I am keen on programming, because it helps them to think about how to solve problems, if handled correctly it can get them to think in a logical way.

Callum explained how he tried to get students to think in a logical way:

I want them to pull back a bit before they tinker, a bit more reflection back on the criteria, for example "Could you improve the process, design is a critical element?" We need to teach and develop the design process more, and avoid tinkering and doing. Flow charting is compulsory. I make the students show me a flow chart before they start programming. The intention of the use of the flow chart was to get them to think logically about what they would do, especially the software. But I expected them to use almost trial and error in getting the software to do what the flow chart intended.

This explanation illustrates Callum's rules for the use of the flow chart tool in achieving his intended learning objectives. Classroom observations confirmed the emphasis he placed on flow chart use. When scaffolding students through programming problems he generally requested to see their flow chart and asked students to explain their objectives before attending to the details of scaffolding their programming. Callum paid scant heed to the physical construction of students' robots.

There was considerable evidence that Callum saw robotics as an instrument to teach logical thinking rather than specific scientific concepts, but he said that he saw potential for robotics to be used as a context to teach physical science. Despite the fact that the unit was part of the science course, he did not, however intend to attempt to teach science content through the activities, justifying his stance as follows:

About a third of the class have a reasonable background in physical and natural science. On the other hand, two thirds of the class had only done basic foundation science one, so with this difference in backgrounds it would be hard to integrate science.

When asked if he used "at the moment" opportunities to extract science principles he responded "It does not happen." This was his acknowledgement that he did not focus on making the underlying science principles explicit, rather his priority was on developing generic problem solving skills.

Callum was "against letting them copy or download programs from the web," indicating that he wanted students to create their own solutions. He explained his beliefs on the role of the teacher in facilitating learning, and the kinds of scaffolding that were appropriate as follows:

I don't lead them by the hand, I don't give these guys parts of programs or whatever, I don't want to do that, I let them struggle, I give them technical help and I explain what the icons do and I help them debug, but I leave the thinking pretty much to them.

Callum saw the division of labour (See Figure 1) with himself as the primary source of technical help, but that it was the students' responsibility to develop the logic sequences to produce the programs. His technical scaffolding is typified by the way in which he discussed a problem that a girl had with programming her robot to recognise the black line of the circle circumference. The RCX brick can be programmed to respond to various light thresholds, hence, the numbers in the following evidence sample indicate the light intensity sensed by the light sensor, (a lower number indicating less intense light). 
Callum: So what is it looking for? What did we see when we tested the brightness level of the light?

Cassy: That it wasn't very bright at all.

Callum: That it was 54, as soon as it starts running it detects that it is less than 55 , so it does a switch-a-roo on the engines for a second. That is why it shuttered. OK, how to modify it?

Cassy: How much? Eighty something?

Callum: No, what were the levels when I hit the blue button?

Cassy: Umm?

Callum: That currently says forty-six. The dark line was 35 and the white line was 54, so what should we be looking for?

Cassy: Forty?

Callum: Ok, somewhere in the middle, but before we do that let us look at the logic of your program what exactly are you expecting your robot to do?

While the details of the effect of student activity are described in subsequent assertions a brief summary of the interactions between Callum's beliefs, goals, the rules he implemented and the consequential divisions of labour in the class follows. Since Callum believed that students ought to construct their knowledge he attempted to work from the students' current knowledge point and assisted them to progress by giving them technical advice via questioning in order to help them reach their own conclusions. The division of labour was such that Callum mostly facilitated in the context of individual or small group discussion and rarely did he structure whole class discussions, thus students spent almost all of the lessons in small group engagement that resulted in inquiry use of instruments. The objects (learning outcomes) of the activity reflected Callum's management of the classroom community. There was a diversity of robot chassis and program solutions including strategies to find the cans reflecting a range of student problem solving endeavours. Two groups (not focus groups) failed in the practical task of pushing the cans from the circle.

\section{George}

George articulated his beliefs about what was a priority for learning outcomes as follows:

(Students should) be able to work quite independently without any direction from me and come up with solutions to the challenges that we give them...but they are not mature adults, they are kids and you know very dependent. I want them to develop planning and design and the thinking that goes on.

These goals focus on conceptualisation and generic cognitive skills. He indicated that students needed guidance to become independent learners. George believed that covering what he identified as 'the basics,' was essential and for this reason he gave students the basis of a program solution in the initial phase of learning activities:

\footnotetext{
I give them a basic program and they have to amend it. They need structuring or you will have period after period where they flounder. So I give them segments of coding, in part, because I want them to see the higher level of programming. You see our groups are spread in ability and they need structuring or you will have period after period where they flounder, which is not my style. I struggle with the idea of openness and just letting students experiment.
}

This statement begins to illustrate George's rationale for rules for the use of instruments (mediating artefacts- see Figure 1) by students. In order to avoid the problem of students struggling with programming, George gave students programs he had previously written for them to amend. The intent of this strategy was to enable 
them to progress more quickly to advanced programming. Regarding the development of creativity George believed "creativity will come if the basics are covered."

The students were required to do flow charts as part of the planning process. However, George did not insist that these be constructed prior to the beginning of building and programming. In scaffolding his students he did not take account of their flow chart constructions.

George's goals for the students were reflected in the division of labour (Figure 1) within the community. It was his responsibility to provide detail and structure and the responsibility of the students to master the basics. George did not emphasise the potential to extract the science embedded in the technology based activities stating "One thing we have not done well so far is bringing in the scientific concepts, gearing, torque and those good physics ideas." George provided a rationale for this situation:

It is the computer that is driving it, this course is like a maths computing course. I just happened to be using that computer in a technical design. We are not bringing the science out or the technical principles.

This was recognition of the powerful role the Labview program tool had in influencing the nature of the learning community as reflected in general classroom activity and even upon his own goals and the rules he enacted in the classroom.

Consistent with his beliefs George felt it was his task to provide a high degree of structure, certainty, and to be the source of information and to provide materials and information to facilitate student success (division of labour- see Figure 1). This was reflected in the highly structured way he started and finished lessons with mini lectures and in the directive nature of his scaffolding of individual students during group work as illustrated below.

Right, try it again, nice and slowly, you have got to find where the little green exit button comes out on your wiring tool. Run it up to motor C. There! No it is over here, see isn't it? Watch me, come around and watch me. See the little output there? See how the green comes on? Wire it up to that. You have got to go green to green and blue to blue. Now you try it.

At about half way through the study George provided students with a "hints file" that modelled a successful program for finding and removing the cans. Prior to this the students were having some difficulties developing a range of different approaches both in terms of strategy and implementation. However, after George's model programs became available, the students adopted his problem solutions. Similarly, George also took primary responsibility for the robot chassis design by supplying them with an exemplar model chassis in the third week. Subsequently, all but two of the eight groups adopted this model. All his students ultimately succeeded in developing robots that accomplished the tasks, however, the products and processes that students engaged in were similar. In summary, George stated a belief in developing planning, design and thinking skills and that learning the basics was central to achieving these abilities. He also believed that it was important that students not be left to "flounder". To this end, the rules for conducting the class and the divisions of labour enacted in the class were such that ultimately George did most of the problem solving and the students modified and implemented his creativity.

In Assertion 1, evidence was presented to illustrate that teachers' differing beliefs found expression in the rules and divisions of labour they implemented and this was reflected in class activity. In the following assertions a finer analysis of how enacted rules, in 
particular those associated with tool use were seen to interact to influence class activity and learning outcomes.

Assertion 2: More holistic problem solving approaches were associated with students' systemic use of flow charting.

The planning of the projects was an integral part of the learning cycle. Both teachers taught the students the mechanics of flow chart construction and the students were required to submit their plans and flow charts upon completion of the project for the purposes of assessment. However, as noted in Assertion 1, Callum placed high priority upon students' use of the flow chart tool while George did not.

It was observed that two groups (Groups 1 and 2) in Callum's class conjointly developed a flow chart to plan their computer program and found them useful. For example, Laurie (Group 1), who did most of the translation of the flow chart into the computer program in his group, commented:

It is much easier to work from a flow chart, it is much easier, it is all set up

logically and you just use the icons, see what it has to do, and make it do the job.

Laurie (Group 1) could program a problem solving strategy for the robot very quickly. He used a flow chart and was able to translate the logic of the flow chart into an icon based program to instruct the robot to complete the tasks. Laurie's comment suggested that the flow chart enabled him to compartmentalise the problems, thus reducing his cognitive load.

Amy (Group 2) commented that the flow charts were useful depending on the complexity of the task, "not so much in the beginning but as you advance it gets easier with the flow chart...just planning it out," and added:

They were a good idea, but yes, they were good for the bigger and more advanced things rather. But with the simpler things like running it for 5 seconds forward and back, I don't think they were really good .... Yeah, for more complex things they make it a lot easier.

It seemed that the flow chart was a useful tool for guiding programming for the students in these groups, and facilitated the construction of advanced programs in some instances. These students did not make radical changes to their processes and products (Objects- Figure 1). It is suggested that students who made more discrete changes in their programs and constructed robots could be identified as being more capable problem solvers (e.g., Groups 1 and 2 in particular).

In contrast, the remainder of the students in Callum's class and all those in George's class constructed their flow charts after they had constructed their programs rather than as a planning tool to assist in the completion of the task. It may be that the students considered that planning occurred at the moment of innovation and prior construction of a flow chart did not coincide with their innovative design. Wil, (Group 3) a Year 8 student summed up the prevalent rationale for this sequence of labour:

The flow charts were a waste of time, most people actually just think up the program in their head and use trial and error to fix it, they do not worry about some flow chart like that, you never take notice of it when you're designing a program, most people just say, oh well it's a flow chart, we've done it, lets do the programming our way and not worry about the flow chart.

Sylvester (Group 3) supported these sentiments: 
I agree with Terrance and Wil because when you do it like you have a program in your head and when you go and do a flow chart it sort of confuses you more, like you have a worse chance of getting it right than you did before you did it.

These comments were supported by Chantelle (Group 6), a Year 10 student, who simply noted; "You can’t really follow a flow chart; it just establishes what to do."

Resistance to the flow chart tool appeared to be based on two premises, firstly as illustrated by the comments of Wil, the flow chart construction did not mirror student thinking and problem solving processes. So they simply did a flow chart then ignored it and problem solved in their own way. Secondly, as articulated by Sylvester, not only was the act of flow chart construction a process not natural to the way they thought, but the disjunction was so serious it acted to confuse their thinking.

Neglecting to use the flow chart tool effected their object (robot construction - see Figure 1). Groups 3 and 4 began construction from scratch several times. For example, when they came to a problem that they found insurmountable they abandoned their approach and started again from the beginning, again without the use of a flow chart. Both Wil's group (Group 3) and Matt's group (Group 4) had "torn up their program and started anew" (Wil). Matt noted:

\footnotetext{
Oh the one (program) we are on now, three times now we have had to completely

demolish our program and start it again. One time it was just way to big, the other two times it just stopped working, it was not the batteries, it just stopped working.
}

Interestingly, without the guidance of a flow chart, usually, each iteration was similar to the last and each time Matt came to a critical problem similar to the last program. Ultimately Matt copied a program that his teacher had displayed on the whiteboard.

The two groups that used the flow chart cognitive tool enacted strategic and holistic solutions to solve their problems. In contrast, those groups who did not plan with flow charts tended to rely more on chance for the robot to "find" the cans to be pushed out.

The discourse below illustrates that the Group 1 were continually micro-planning the robot to perform strategically. In this example they were discussing how to optimise the robot's search pattern:

\footnotetext{
Michael: I think we should do star (pattern) thing because it gets most ...it will go like there and there. It will get the whole... we have to (get the robot to) draw a star.

Ron: We should have put a pencil on the back of the robot to see what it draws.

Teacher: Except you don't want the same star. You've got to draw it so ...you can get star patterns that are completely repetitive.

Ron: $\quad$ Ours won't be repetitive because we have touch sensors, it hits a can it will turn to it and change the pattern.
}

Clearly, Ron's group was well aware of planning to optimise the robot's chances of finding a can to push out.

The robot had been programmed so that when a touch sensor on the robot came in contact with a can, the robot would turn about ten degrees towards the can and begin to push the can from a centre-mounted pushing bar base and push the can out of the circle. The group had experimented with the height of the pushing bar and determined a height that would not push the can over, thus avoiding the potential of it rolling away from the robot. Ron was aware that the turn initiated by the touch sensors would 
prevent the star pattern becoming repetitive, that is, it would introduce variation and thus the chances of blind spots in the search pattern remaining would be reduced greatly. This group had carefully planned the turn of the robot so that when the light sensor detected that the robot had crossed the black line of the circle boundary, the robot would reverse, then turn to not come back on the previous trajectory which would mean a turn of 180 degrees, but turn about 160 degrees to create the star pattern. Their robot never exited the circle and moved away from the trial area without returning. Their approach was holistic in that it accounted for programming and construction in a strategic way. Discourse indicated that during the refinement phases the three group members collaborated in trialling and refining the robot and its actions. The approach to the problem was both strategic and holistic. Similarly, group 2 developed an integrated solution such that their robust robot covered the circle; however, they appeared to be less aware of the importance of variation in a search pattern.

In contrast to the strategic thinking of Group 1 and to a lessor degree Group 2 the remaining groups tended to tinker and appeared to be less aware of cause and effect as described by Dale (Group 4):

When the motors do not work properly we found we had to keep switching the little chord (wiring from the RCX computer to the motors) around because having the chords both ways at the same time made the motors not run properly, so we had to turn one of them around so that both motors would run forward. We do not really know why, but we got a bit annoyed at that and thought about how we could change it.

Group 5 established no search pattern as such, that is they and the remaining groups were not observed to plan optimisation in searching for cans. Cass (Group 5) described her search strategy:

I'm just counting on it going basically forward, sense the black line, back off, turn a bit, go forward and then it will hit the black line and keep coming around and around and around.

Observations of the robot's movements confirmed that there was no planned search pattern, the movement of the robot within the circle was apparently random. Cass remarked, "It is a beast, it just goes (its own way)," and she was unable to articulate why she had programmed it that way. Because the group did not calibrate the turn correctly, during trials the robot frequently "got lost" and exited the circle, unable to return on its own accord. The response of the robot to the light sensor's activation as it crossed the black line of the circle boundary was not linked to an appropriate reversing and turning response by the robot to keep it within the circle.

The interaction between robot construction and program was evident for all groups, however, the level of interaction varied for different students. A change in the structure of the robot, for example, the use of different size wheels, different gears, different mechanisms of steering, location of sensors, or addition of sensors caused the robot to function differently and the students usually modified the software program to account for these changes. Likewise, changes in the program such as motor power levels and motor operating times sometimes prompted students to alter the robot gears or chassis length. The extent to which students accounted for the interactions between Labview and robot construction varied between groups.

Groups 1 and 2 were meticulous in both identifying critical features of the problem and only changing one variable at a time. When Laurie (Group 1) and his group were refining their search pattern for the cans they worked very systematically. 
$\begin{array}{ll}\begin{array}{l}\text { Laurie: } \\ \text { Researcher: }\end{array} & \begin{array}{l}\text { We are just changing how it turns...we just change the time the robot turns. } \\ \text { Mike ehaet: }\end{array} \\ \begin{array}{l}\text { Laurie: } \\ \text { Researcher: }\end{array} & \text { About } 200 . \\ \text { Laurie: } & \text { Have you any idea what pattern it is going to make. }\end{array}$

In testing this pattern, Mike (Group 1) would trace the path of the robot with his finger, and the entire group would then go back to the program and modify it. The following discourse was typical of their problem solving as they manipulated the time one motor operated in a forward direction and the other motor operated in the reverse direction in order to have their track driven robot turn:

$\begin{array}{ll}\text { Mike : } & \text { That's a fast turn. } \\ \text { Ron: } & \text { It still turns too much. } \\ \text { Mike: } & \text { No I think that is good. It backs off a way, that is good. } \\ \text { Researcher: } & \text { Explain to me why you guys made it reverse out of the circle before } \\ & \text { it came in again. } \\ \text { Mike: } & \text { So that when it is turning, these (the feelers for the touch sensors) do } \\ & \text { not get caught on the cans. } \\ \text { Laurie: } & \text { It goes ...it hits the line about there, at about close to } 90 \text { degrees. } \\ \text { Mike: } & \text { Can I have a go?...Run it on the line...it is about } 2 \ldots 2 \text { hundred and } \\ & \text { something so it is turning too much. }\end{array}$

The vignette helps the reader to appreciate that these students were generally accounting for one variable (e.g., a turn) at a time. In the case of Mike's group, they were considering the effect of that variable, or feature, on a number of robotic behaviours.

The final program and robot of Group 1 did not fail in about 10 trials. The robot was robustly built and geared for power; they had chosen strength over speed. They made the decision to gear their robot down and use a track system after a prototype failed to push two cans simultaneously. Their final robot could push up to three cans at once, something that occasionally happened when the teacher clumped the placement of cans. As a result of its low gearing the robot was relatively slow and to account for this the group focused on developing an effective search pattern and using feelers. That is, they integrated the physical and programming capacity into their solution. Amy's group (Group 2) were also observed to be systematic in their approach, changing one variable at a time to understand clearly the effect of change in the computer program on the robot actions. The data indicated that the two groups who used the flow chart cognitive tool were aware of the interactions between programming and construction and took these interactions into account when changing either the program or the structure of various systems in the robot.

However, among the focus groups that did not use flow charting (Groups 3, 4, 5 and 6) the connection between programming changes and robot behaviour was ill defined. The critical aspect of this behaviour was changing more than one variable of the robot at a time. For example, Cass (Group 5) and her group were working on getting their robot to turn more, so Cass would increase the time for one motor to stop and the other to engage. However, the builder (Steph) simultaneously lengthened the wheelbase, thus making the torque required to turn the robot greater, which nullified the program change. Similarly, when confronted with a turn problem, Matt (Group 4) changed the operating time of one engine and not the other. At the same time, Bill the builder changed the gearing. This changing of more than one feature at a time is akin to not controlling variables, but it was not limited to a disjunction between 
programming and construction. Wil (Group 3) was observed to alter both the power level and the time for running the motors simultaneously. When confronted with a similar problem of turning, Matt (Group 4) changed the duration of running of one engine in the program and not the other, not realising that Bowen (Group 4) had changed the gearing ratio on the motors which interfered with the effect of the programming change. In this way student focus was on the individual parts rather than the sum of the parts. Thus, there was evidence that those groups that did not use the flow chart were less methodical in their approaches and used trial and error strategies more so than did the two groups that used the flow charting tool, and did not account for the nexus between programming and construction effects. For groups 3, 4, 5, and 6 the connection between programming changes and robot behaviour was more ill defined and their solutions less strategic.

\section{Discussion and conclusions}

Activity theory provided a useful framework for analysing and interpreting classroom interactions and events and enabled the researchers firstly, to determine how teachers' beliefs influencing pedagogical practices and outcomes and, secondly, conduct a finegrained analysis of middle school students' approaches to problem solving, designing, and construction in a robotics unit. The key findings are presented in the form of assertions and related discussion.

The findings demonstrate that teachers' beliefs and goals did lead to the development of contrasting learning environments and learning communities in the two classrooms (Assertion 1). For example, the nature of scaffolding that George provided tended to encourage students to replicate his solutions, both in relation to construction and programming, and to forgo their own solutions. This contrasted with the diversity of design that emerged in Callum's class. In this regard the study confirms earlier findings on the relationship between teachers' beliefs and pedagogy (e.g., Alexander, 1996; Thompson, 1992) and the effect of pedagogy upon student learning of mathematics (e.g., Skemp, 1978; Thompson, 1992) and technology (Anning, 1994). This study differs from previous studies in that the mechanisms by which beliefs and goals found expression through rules and for instrument use and division of labour (Figure 1) is made explicit.

Davyov (1999) noted that activity is related to the process of transformation. In this study the transformation of most interest is the changes that might occur in the minds of the students, these changes were the principal objects, but were reflected in concrete manifestations. Thus the term object has a dual character, a tactile as well as a cognitive manifestation. There were two main indicators of learning outcomes in our study, what the students built and what the students did and said. This view that the artefacts are one manifestation of the object is supported by Tolman (1999) who described the development of a child's activity in terms of its concrete conditions of one's life, the concrete not being limited to tactile products but also referring to the social nature of the individual including his or her thoughts and interactions. The results of this study indicate that where the teacher provided explicit scaffolding, (George) internalisation or the reproduction of culture was most uniform and functional, that is, the robots worked, culture was successfully replicated but little student creativity was demonstrated, hence externalisation was limited. This study shows that greater educational transformation, in particular externalisation and the creation of new learning objects, of fresh student creativity and new creation was 
more evident in the class were specific guidance was less, but support of problem solving via the use of flow charting was greater.

The contrasting teachers' beliefs, practices and resultant learning environments and learning communities did appear to influence student activity. In general, the construction and programming of robots was viewed by most students as an authentic challenge as evidenced by the degree of commitment to the task and the quality of the discourse. Clearly, the instruments (mediating artefacts - tools) helped to foster communities of learning at the micro level (Figure 1) that produced educational objects including learning outcomes associated with design technology processes such as planning, constructing and evaluating artefacts. In this regard the study supports previous findings (e.g., Bergen, 2001; Levien \& Rochefort, 2002; Mauch, 2001) that Lego robotics can be a valuable educational tool for teaching problem solving and design. This was most evident in the very integrated and holistic problem solutions developed by some groups, in particular, Groups 1 and 2 that we suggest could be ascribed to student's systematic use of flow charting (Assertion 2). It was evident that the activities gave all students the opportunity to work scientifically, that is to employ the mental processes, routines and protocols that are the essence of scientific enterprise and "to make sense of phenomena, to investigate, understand and communicate” (Queensland Schools Curriculum Council, 1999, p. 1).

Perhaps more so in a technology rich learning environment such as Lego Robotics the instruments or mediating artefacts (flow charts, Labview, RCX computer brick and Lego construction pieces) dominated the classroom discourse and had the effect of spreading the cognitive quality of student endeavour. Evidence for this was seen in the dispersed quality of student outcomes, some robots being simple in design and some being very complex, well planned and well constructed with a good match between the robot and the program that controlled it.

The quality of student discourse also varied considerably with Groups 1 and 2 recorded as engaging in discussions that accounted systematically for variables while in other groups, strategy was often closely aligned to tinkering and trial and error. The range of student activity and object outcomes was greater in Callum's class. Given his objective of wishing to put the responsibility for creative effort on to the students, this is hardly surprising. In Callum's class the division of labour fostered by him placed students in the roles of designers and creators and potentially generated a rich metacognitive context for discussion including analysis of the relevant science concepts. However, the enactment of division of labour and rules meant that the forum for discussion was not used to achieve whole class discussion at a macro level. Without such discussion it is difficult to see how either class as a whole could form an integrated learning community where the science underpinning the activities could be made explicit, a problem acknowledged by both teachers. Two groups did demonstrate advanced capabilities for problem solving, yet the distribution of their expertise and understandings for the benefit of other less capable groups was not attempted at a macro level.

The planning instrument (mediating artefacts-tool) flow charting was used differently by different groups and this deviation influenced the way students worked and ultimately the artefacts and degrees of internalisation and externalisation. The findings of this study indicate the use of flow charting influenced students activity in a number of ways. Firstly, some students found that the use of the flow chart tool assisted their logic expression, consistent with previous findings (e.g., Crews \& 
Butterfield, 2003; Satchwell, 1997). In contrast, other students felt that the use of the flow chart instrument was either irrelevant or even in some instances an obstacle to finding problem solutions. In essence, these students stated that the use of flow charts was not in alignment with their natural way of thinking. Secondly, the use of the flow chart instrument was associated with consistency in approach. Those students who embraced the flow chart instrument tended to persist with a particular problem solving approach, with the use of flow chart giving coherence and stability to their thinking. Students who rejected the use of flow charts were more inclined to tear up their initial solutions and start afresh, sometimes with radically different approaches, sometimes with almost identical approaches. Satchwell (1997) noted that the use of flow charts facilitated conceptual chunking and helped students to gain an overall understanding of the process involved in the problem solutions. The finding that those students who used flow charts persisted with particular approaches, perhaps because these were coherent for the students while those who abandoned their approaches had less holistic understandings of their strategies, is consistent with the view put forward by Satchwell (1997).

Abandonment of problem solving approaches was particularly evident when the teacher offered a ready alternative. It is likely that the rules for flow chart use enacted by Callum (Assertion 1) were at least partly responsible for Groups 1 and 2 developing the habit of using flow charts in their planning and problem solving. It can be claimed that because they were supported in the use of the flow chart instruments these students acquired an appreciation of its potential to assist their thinking. Despite this, the majority of students in both classes resisted its use. Clearly, the task of encouraging students to use planning instruments such as flow charts and determining the relationships between the use of planning instruments, creativity and persistence needs further investigation.

In addition to the use of the planning instrument of flow charting, the use of the Labview programming instrument and the use of Lego construction tools were central to the community endeavour. In fact, the typical tools of classroom learning, books, pencils, white boards and desks played a minimal role in the activity of these classes. As noted previously (eg., McRobbbie et al., 2003) the activity of programming tended to dominate the way students worked, that is, students spent more time and more activity on programming compared with construction. However, data presented in Assertion 2 indicated that Groups 1 and 2 in particular, worked in a way that illustrated they better understood the interactions between the program and the physical manifestation and actions of the robot. In addition, these students worked in a more scientific manner, identifying variables and accounting for them individually thus reducing the confounding effects of multiple interacting variables. In contrast, other students (Groups 3, 4, 5, and 6) who resisted the use of flow charts, changed a number of variables simultaneously, thus creating interactions between the program and the physical construction which were difficult to evaluate and account for. On occasions some of these students programmed and constructed in isolation, in that the programmer and the constructor did not collaborate, illustrating a breakdown in the community learning rules. The role of the use of flow charts in facilitating student understanding of the interrelated nature of the learning environment is not clear, however, Satchwell (1997) noted that the use of flow charts enhanced students' holistic views on how systems worked, which is in accord with the data presented in this study. 
If, as Tolmin (1999) stated humans define themselves through the things they create, the use of such tools in middle schools warrant further investigation because in this study the planing tools have been associated with higher quality creations and greater student autonomy, creativity, strategic thinking and metacognitive thinking for approximately one third of the students in the six focus groups. These findings have implications for guiding teachers in the use of sophisticated instruments in technology rich learning environments. Thus, it is recommended that in planning to use Lego robotics kits in the manner described in this paper, teachers should identify specific science outcomes and capitalise on critical learning opportunities that present themselves during lessons to help students make science principles explicit. This should include giving students opportunities to meaningfully discuss the activities in the context of science. In addition, student planning, such as that facilitated through the use of flow chart instruments be actively encouraged and scaffolded so that students can appreciate the potential of flow charts to facilitate their problem solving capabilities. Some teachers may benefit from reflecting on the divisions of labour within the class, in particular the degree to which they assume the creative and problem solving role by providing explicit instruction.

\section{References}

Alexander, P. A. (1996). The past, present, and future of knowledge research: A reexamination of the role of knowledge in learning and instruction. Educational Psychologist, 31(2), 89-92.

American Association for the Advancement of Science. (1993). Benchmarks for science literacy: Project 2061. New York: Oxford University Press.

Anning, A. (1994). Technological capability in the primary classroom. Report Published by University of Leeds, School of Education, Education for Capability Research Group.

Bergen, D. (2001). Learning in the robotic world: Active or reactive? Childhood Education, 77(4), 249-250.

Crews, T., \& Butterfield. J., (2003). Improving the learning environment in beginning programming classes: An experiment in gender equity. Journal of Information Systems Education, 14(1), 69-76.

Davydov, V. (1999). The content and unsolved problems of activity theory. In Y. Engestrom, R. Miettinen, \& R. Punamaki (Eds.), Perspectives on activity theory (pp. 39-53). Cambridge, UK: University Press.

Deek, F., Hiltz, S.R., Kimmel, H., \& Rotter, N. (1999). Cognitive assessment of students' problem solving and program development skills. Journal of Engineering Education, 88(3) 317- 326.

Education Queensland. (2001). New basics project: Technical paper.

$\begin{array}{llll}\text { Retrieved July } & 1, & \text { from }\end{array}$ http://www.education.qut.edu.au/nortonsj/Curriculum/New-Basics-paper.rtf $(1 / 07 / 03)$

Engstrom, Y., \& Miettinen, R. (1999). Introduction. In Y. Engestrom, R. Miettinen, \& R. Punamaki (Eds.), Perspectives on activity theory (pp. 1-18). Cambridge, UK: Cambridge University Press. 
Erickson, F. (1998). Qualitative research methods for science education. In B. J. Fraser \& K. G. Tobin (Eds.), International handbook of science education (Part 2, pp. 1155-1173). . Dordrecht, The Netherlands: Kluwer.

Leontyev, A. N. (1977). Activity and consciousness: Philosophy in the USSR, problems of dialectical materialism: Progress Publishers. Retrieved February 17, 2004, from http://www.marxists.org/archive/leontev/works/1977/leon1977.htm

Levien, K., \& Rochefort, W. (2002). Lessons with LEGO - Engaging students in engineering courses. In Proceedings of the 2002 American Society for Engineering Education Annual Conference and Exposition, Oregon State University, Oregon., American Society for Engineering Education.

Lincoln, Y. S., \& Guba, E. G. (2000). Paradigmatic controversies, contradictions, and emerging confluences. In N. K. Denzin \& Y. S. Lincoln (Eds.), Handbook of qualitative research (2nd ed., pp. 163-188). Thousand Oaks, CA: Sage.

McRobbie, C. J., Norton, S. J., \& Ginns, I. S. (2003, April). Student designing in a robotics classroom. Paper presented at the annual meeting of the American Educational Research Association, Chicago, IL.

Mauch, E. (2001). Using technological innovation to improve the problem-solving skills of middle school students: Educators' Experiences with the LEGO Mindstorms Robotic Invention System. Clearing House, 74(4), 211-214.

National Advisory Committee on Creative and Cultural Education (NACCCE). (1998). All or futures: Creativity, culture and education. Nottingham, UK: DfEE Publications.

Noble, M. (2001). The educational impact of LEGO Dacta materials. Sheffield Hallam University. Retrieved November 2, 2004 from, http://www.lego.com/education/download/sheffield_hallam.doc.

Oxman, R. (1999). Educating the designerly thinker. Design Studies, 20(2), 105-122.

Papert, S., \& Harel, I. (1991). Situated constructivism. Retrieved February 21, 2002. From http://www.papert.com/articles/SituatingConstructionism.html

Queensland School Curriculum Council. (1999). Science Years 1 to 10 Syllabus. Brisbane, Queensland: The Office of the Queensland School Curriculum Council.

Queensland Studies Authority. (2003). Technology: Years 1-10 syllabus. Brisbane, Queensland: The State of Queensland. Retrieved February 10, 2004 from, http://www.qsa.qld.edu.au/yrs1_10/kla/technology/index.html.

Queensland Studies Authority. (2004). Mathematics Years 1 to 10 Syllabus. Retrieved September 18, 2003, from, http://www.qsa.qld.edu.au/yrs1_10/kla/mathematics/index.html.

Roth, W. (2001). Modelling design as situated and distributed. Learning and Instruction, 11(2), 11-239.

Roth, W., Tobin, K. Zimmerman, A., Natasia, B., \& Davis, C. (2002). Lessons on and from the dihybrid cross: An activity-theoretical study of learning in co-teaching. Journal of Research in Science Teaching, 39(3), 253-282. 
Satchwell, R. (1997). Using functional flow diagrams to enhance technical systems understanding. Journal of Industrial Teacher Education, 34(2), 1-28. Available http://scholar.lib.vt.edu/ejournals/JITE/v34n2/Satchwell.html.

Skemp, R. R. (1978). Relational understanding and instrumental understanding. Arithmetic Teacher, 26(3), 9-15.

Thompson, A. G. (1992). Teachers' beliefs and conceptions: A synthesis of the research.

D. A. Grouws (Ed.), Handbook of research on mathematics teaching and learning (pp. 127-146). New York: National Council of Teachers of Mathematics.

Tolman, C. (1999). Society versus context in individual development: Does theory make a difference? In Y. Engestrom., R. Miettinen, \& R. Punamaki (Eds.), Perspectives on activity theory (pp. 70-86). Cambridge, UK: Cambridge University Press. 